\title{
Cough and asthma diagnosis: physicians' diagnosis and treatment of patients complaining of acute, subacute and chronic cough in rural areas of Japan
}

This article was published in the following Dove Press journal:

International Journal of General Medicine

26 February 2010

Number of times this article has been viewed

\author{
Akira Yamasaki' \\ Keichi Hanaki \\ Katsuyuki Tomita ${ }^{3}$ \\ Masanari Watanabe \\ Yasuyuki Hasagawa' \\ Ryota Okazaki ${ }^{i}$ \\ Miki Yamamura' \\ Kouji Fukutani ${ }^{4}$ \\ Yuji Sugimoto 5 \\ Kazuhiro Kato ${ }^{4}$ \\ Masahiro Kodani ${ }^{6}$ \\ Toshikazu Ikeda ${ }^{7}$ \\ Tatsuya Konishi ${ }^{8}$ \\ Yuji Kawasaki ${ }^{9}$ \\ Hirokazu Tokuyasu9 \\ Hiroki Yajima ${ }^{3}$ \\ Hitoshi Sejima10 \\ Takeshi Isobe"I \\ Eiji Shimizu' \\ SAN-IN Asthma Research \\ Group \\ 'Third Department of Internal Medicine, \\ ${ }^{2}$ Department of Pediatrics, Tottori \\ University, Japan; ${ }^{3}$ Department of \\ Respiratory Medicine, Hakuai Hospital, \\ Japan; ${ }^{4}$ Department of Respiratory \\ Medicine, San-in Rosai Hospital, Japan; \\ ${ }^{5}$ Department of Respiratory Medicine, \\ Tottori Prefectural Central Hospital, Japan; \\ ${ }^{6}$ Department of Respiratory Medicine, \\ Tottori Red Cross Hospital, Japan; \\ ${ }^{7}$ Department of Respiratory Medicine, \\ National Organization, Matsue Hospital, \\ Japan; ${ }^{8}$ Department of Respiratory \\ Medicine, Matsue City Hospital, Japan; \\ 'Department of Respiratory Medicine, \\ Matsue Red Cross Hospital, Japan; \\ ${ }^{10}$ Department of Pediatrics, "Department \\ of Internal Medicine, Shimane University, \\ Shimane, Japan
}

Correspondence: Akira Yamasaki

Division of Medical Oncology and

Molecular Respirology, Department

of Multidisciplinary Internal Medicine,

Faculty of Medicine, Tottori University,

36-I Nishi-chou, Yonago, 683-8504, Japan

Tel +8I 859386537

Fax +8I 859386539

Email yamasaki@grape.med.tottori-u.ac.jp
Background: Cough is one of the most common reasons for visiting a clinic. The causes of cough differ according to the duration of cough. Infectious disease is commonly observed in acute cough while noninfectious disease is commonly observed in chronic cough. On the other hand, cough is frequently observed in patients with asthma/cough variant asthma (CVA).

Objective: In this study, we investigated the causes of cough in a rural region in Japan and the clinical examination and treatment for the patients diagnosed as asthma/CVA.

Methods: We analyzed 124 patients who complained of cough.

Results: The most common reason for acute cough was respiratory tract infection while asthma/CVA is the most common reason for subacute and chronic cough. The diagnostic procedure for asthma/CVA depends on clinical symptoms in asthmatic patients with acute cough. While in asthmatic patients with subacute and chronic cough, diagnosis of asthma depends on clinical examinations including chest radiogram, immunoglobulin E, white blood cells counts, sputum examination or spirometry as well as symptoms. For the treatment of asthma, the use of long-acting $\beta_{2}$-stimulant was dominant in asthmatic patients with acute cough while the use of leukotriene receptor and inhaled corticosteroid were dominant in asthmatic patients with subacute or chronic cough.

Conclusions: Diagnosis and treatment for asthma differs according to the duration of cough. Simple guidelines for asthma/CVA according to the duration of cough might be necessary for diagnosis and treatment of asthma/CVA for general physicians especially in rural areas.

Keywords: cough, diagnostic procedures, cough variant asthma, asthma

\section{Introduction}

Cough is the most frequent morbidity-related reason to visit a physician in the United States, ${ }^{1}$ Great Britain, ${ }^{2}$ Australia, ${ }^{3}$ and Japan. ${ }^{4}$ Several guidelines for diagnosis and management of cough have been established in the United States, ${ }^{5}$ Europe ${ }^{6}$ and Japan. ${ }^{7}$ The guidelines established in Japan categorize cough according to the duration of symptoms. Chronic cough is defined as persistent for more than eight weeks. Subacute cough is defined as persistent for three to eight weeks. Acute cough is defined as persistent for less than three weeks. ${ }^{7}$

The likelihood of disease causing cough varies according to the duration of cough. In acute cough, causes of most cases are infectious diseases such as common cold or subsequent post-infectious cough. In subacute and chronic cough, the ratio of noninfectious diseases is increased for causes of the cough in accordance with the duration of cough. Major causes of chronic cough are bronchial asthma, postnasal drip and gastroesophageal reflex disease (GERD) in the United States. ${ }^{5}$ On the other hand, Fujimura 
and colleagues reported that main causes of chronic cough without any abnormal findings in chest X-ray test are atopic cough, sinobronchial syndrome and cough variant asthma (CVA) in Hokuriku area of Japan. ${ }^{8}$ Similar observations are obtained from other areas of Japan. ${ }^{9,10}$

The diagnostic procedures also affect cough etiology. The diagnosis of bronchial asthma is made based on symptoms such as cough, wheezing in the early morning or midnight, dyspnea, and chest oppression. ${ }^{11}$ A main symptom in patients with CVA is chronic cough. ${ }^{12}$ General examination including chest X-ray test, complete blood counts with eosinophil number, spirometry, and sputum examination might be required for the differential diagnosis of chronic cough, CVA, or bronchial asthma. However, how physicians use these methods for diagnosis of asthma/CVA has not been studied yet. Furthermore, how physicians treat those patients who are diagnosed as asthma/CVA has not been well studied.

In 2003, the 2nd edition of Guideline for Diagnosis and Treatment of Cough was proposed by the Japanese Cough Research Society. ${ }^{7}$ In this guideline, differential diagnosis of chronic cough and diagnosis criteria for CVA, atopic cough, sinobronchial syndrome (SBS), postnasal drip, GERD, psychogenic cough and postinfectious cough are defined. However, it seems difficult for many general clinic physicians to diagnose patients with cough for bronchial asthma or CVA according to these criteria because of lack of equipment for cough provocation test, and spirometry.

In this study, we investigated the causes of acute, subacute and chronic cough in a rural region of Japan (San-in area) and the diagnostic procedure and treatment of patients who are diagnosed with CVA or bronchial asthma in this area.

\section{Methods}

This questionnaire-based study was conducted between April and June 2007 at 19 medical centers and nine clinics in the San-in area of Japan. During this period, 124 patients who visited a doctor for cough were questioned. Therefore, some but not all the patients visited the clinic for the first time for cough. This study was approved by the ethical committee of Tottori University. Informed consent was obtained from all subjects who participated in this study.

To conduct this study, we devised the questionnaire shown in Table 1 (originally described in Japanese). The spirometry, chest X-ray test, sputum eosinophilia, and complete blood count including absolute eosinophil number, and C-reactive protein were measured by each physician. Final diagnoses of acute, subacute, or chronic cough were made by each physician (acute cough being defined as persistent
Table I Questionnaire (originally described in Japanese)

I. How long has it been since you have had cough?
$\square$ within one week $\quad \square$ within two weeks
$\square$ within three weeks $\quad \square$ within one month
$\square$ one to two months $\quad \square$ more than two months
2. Do you have symptoms such as wheezing or chest oppression?
$\square$ Yes $\quad \square$ No

cough for less than three weeks, and subacute cough being defined as cough for more than three weeks but less than eight weeks, and chronic cough being defined as cough for more than eight weeks).

\section{Statistical analysis}

Kruskal-Wallis test analysis was used for comparison of age. Statistical analysis was performed with Graph-Pad Prism 4.0 (Graph Pad Software Inc. San Diego, CA). Differences were considered to be statistically significant at $P<0.05$.

\section{Results}

\section{Characteristics of the patients}

One hundred twenty-four patients visited the clinics complaining of cough. Twenty-five patients suffered acute cough, 45 had subacute cough, and 54 had chronic cough. The average age of the patients with acute cough was the lowest of the three groups, while the average age of the patients with chronic cough was the highest of the three groups. There was statistical difference between the patients with acute cough and chronic cough. The average age of the patients with subacute cough was the second youngest of the three groups and statistical difference was observed between the patients with subacute cough and chronic cough. The number of female patients was larger than that of male in the three groups (Table 2).

Figure 1 shows the age distribution of the patients. The number of patients with acute and subacute cough was larger under the age of 40 years. The number of patients with chronic cough was larger in most of the patients aged over 50 years (Figure 1$)$.

Table 3 shows the diagnosis of cough categorized by duration of cough. In the group of patients with acute cough, the percentage with asthma was $28.0 \%$, while bronchitis

Table 2 Characteristics of patients

\begin{tabular}{lllll}
\hline & Total & Acute & Subacute & Chronic \\
\hline Number of patients & 114 & 25 & $4 I$ & 54 \\
Average age (years) & 50.5 & 44.4 & 45.2 & $57.4^{*}$ \\
Standard deviation & 17.4 & 19.7 & 14.1 & 16.4 \\
Range & $18-74$ & $18-78$ & $20-82$ & $20-84$ \\
Gender (female \%) & 67.5 & 76.0 & 63.4 & 64.8 \\
\hline
\end{tabular}

$* P<0.01$, compared to acute or subacute groups. 


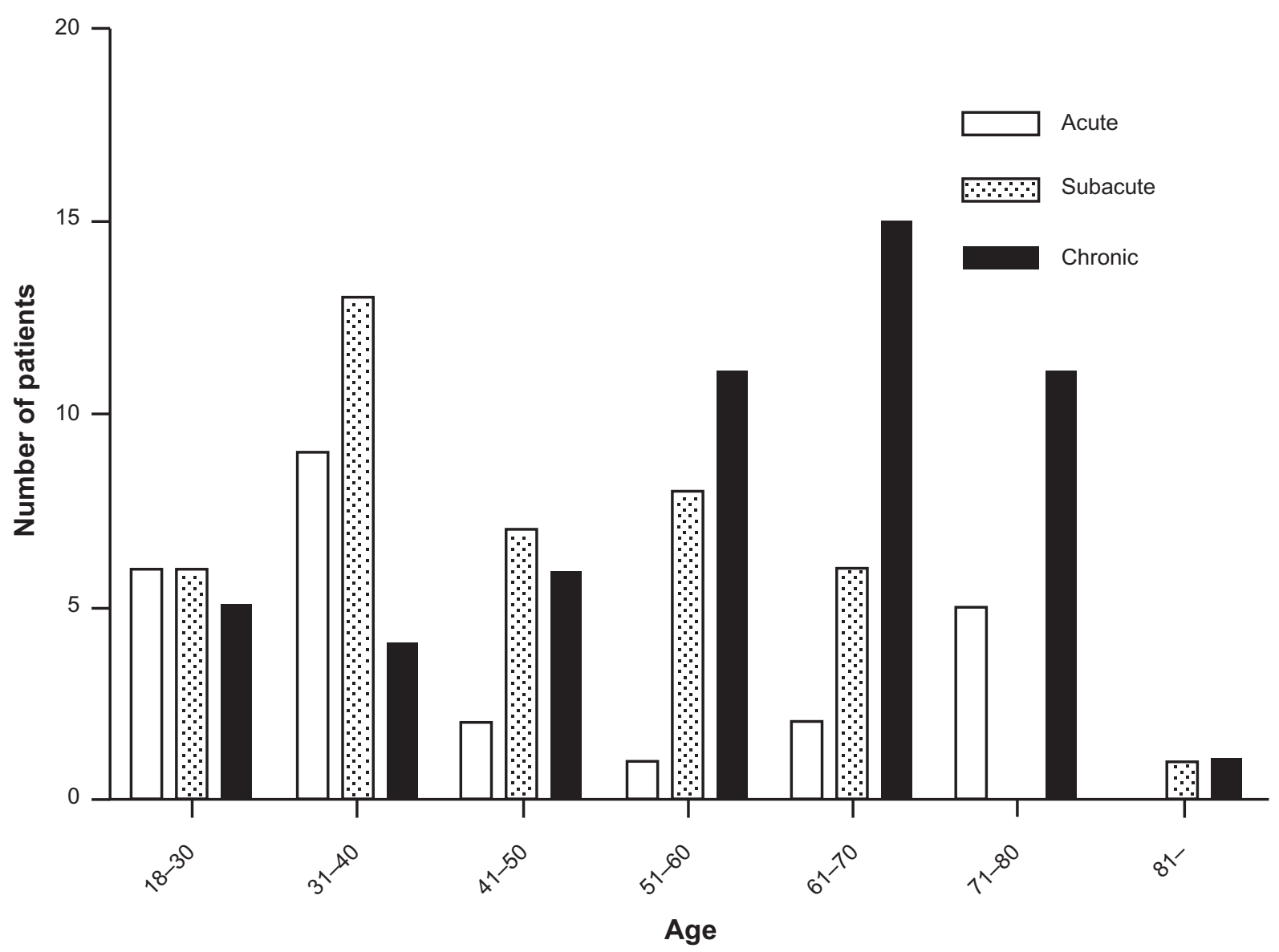

Figure I Distribution of age of patients with cough. The number of patients with acute and subacute cough is larger under the age of 40 years compared to the number of patients with chronic cough. The number of patients with chronic cough was larger compared to the number of patients with acute and subacute cough in most of the generation aged more than 50 years.

caused by respiratory tract infection was $72.0 \%$. In the group of patients with subacute cough, the percentage of asthma was $48.8 \%$, the percentage of CVA is $9.7 \%$, while the percentage of other diseases which are related to microorganism was $7.3 \%$. Other causes were post infectious cough (12.2\%), chronic obstructive pulmonary disease (COPD) (4.8\%), SBS (2.4\%), bronchiolitis associated with rheumatoid arthritis (2.4\%), and causes of unknown origin (12.2\%). In the group with chronic cough, the percentage of asthma was $44.4 \%$; CVA was $9.3 \%$. Other causes were postinfectious cough (11.1\%), COPD (14.8\%), SBS (7.4\%), bronchiolitis associated with rheumatoid arthritis (3.7\%), GERD (4.8\%) and causes of unknown origin $(9.3 \%)$.

In this study, the number of patients with CVA was small. Furthermore, since diagnostic process and treatment of asthma and CVA were similar, we analyzed the diagnosis procedure and treatment for patients with asthma and CVA. Table 4 shows the clinical tests and symptoms for the diagnosis of asthma and CVA. Among patients diagnosed as asthma/CVA in acute cough, chest X-ray test was performed in $57.1 \%$, white blood cell counts with cell differentiation was measured in $42.8 \%$, Immunoglobulin E (IgE) was measured in $14.3 \%$, sputum eosinophilia was examined in $42.8 \%$, and spirometry was performed in $57.1 \%$. The percentage of patients who had typical symptoms of asthma such as wheezing or chest oppression was $85.7 \%$. Among patients diagnosed as asthma/CVA in subacute cough, chest X-ray test was performed in $95.8 \%$, white blood cell counts with cell differentiation was measured in $58.3 \%$, IgE was measured

Table 3 Causes of acute, subacute and chronic cough

\begin{tabular}{llll}
\hline & Acute & Subacute & Chronic \\
\hline Asthma & $28.0 \%$ & $48.8 \%$ & $44.5 \%$ \\
CVA & $0 \%$ & $9.7 \%$ & $9.3 \%$ \\
Respiratory tract infection & $72.0 \%$ & $7.3 \%$ & $0 \%$ \\
Post infectious cough & $0 \%$ & $12.2 \%$ & $11.1 \%$ \\
COPD & $0 \%$ & $4.8 \%$ & $14.8 \%$ \\
SBS & $0 \%$ & $2.4 \%$ & $7.4 \%$ \\
Bronchiolitis associated with RA & $0 \%$ & $2.4 \%$ & $3.7 \%$ \\
GERD & $0 \%$ & $0 \%$ & $4.8 \%$ \\
Unknown & $0 \%$ & $12.2 \%$ & $9.3 \%$ \\
\hline
\end{tabular}


Table 4 Clinical tests and symptoms performed for the diagnosis of asthma/CVA

\begin{tabular}{llll}
\hline & Acute & Subacute & Chronic \\
\hline Chest X-ray test & $57.1 \%$ & $95.8 \%$ & $100.0 \%$ \\
White blood cell count & $42.8 \%$ & $58.3 \%$ & $91.7 \%$ \\
IgE & $14.3 \%$ & $25.0 \%$ & $72.4 \%$ \\
Sputum eosinophil & $42.8 \%$ & $37.5 \%$ & $51.7 \%$ \\
Spirometry & $57.1 \%$ & $70.8 \%$ & $79.3 \%$ \\
Typical symptoms of asthma & $85.7 \%$ & $54.2 \%$ & $82.8 \%$ \\
(wheezing, chest oppression) & & & \\
\hline
\end{tabular}

in $25.0 \%$, sputum eosinophilia was examined in $37.5 \%$, and spirometry was performed in $70.8 \%$. The percentage of patients who had typical symptoms of asthma such as wheezing or chest oppression was $54.2 \%$. Among patients diagnosed as asthma/CVA in chronic cough, chest X-ray test was performed in $100.0 \%$, white blood cell counts with cell differentiation was measured in $91.7 \%$, IgE was measured in $72.4 \%$, sputum eosinophilia was examined in $51.7 \%$, and spirometry was performed in $79.3 \%$. The percentage of the patients who had typical symptoms of asthma such as wheezing or chest oppression was $82.8 \%$.
We next analyzed treatment of asthma/CVA according to the duration of cough. Figure 2 shows the percentage of antiasthmatic drugs used with patients diagnosed as having asthma and CVA. Among patients with acute asthma/CVA, the percentage of inhaled corticosteroid (ICS) use was $42.0 \%$. But the percentage of ICS use increased according to the duration of cough (subacute, $70.0 \%$; chronic, 100\%). A similar trend was observed in leukotriene receptor antagonist (LTRA) use. The percentage of LTRA use increased according to the duration of cough. Among patients with acute cough, use was $30 \%$, with subacute cough, use was $40 \%$, and with chronic cough, use was $60 \%$, while the percentage of long-acting $\beta_{2}$ agonist (LABA) use decreased according to the duration of cough. The percentage of LABA use was $58 \%$ in acute asthma/CVA, $42 \%$ in subacute and $40 \%$ in chronic cough. The percentages of sustained-release theophylline use were highest in chronic asthma/CVA, second highest in acute asthma/CVA and lowest in subacute asthma/CVA patients. Therefore, there was no correlation in use of sustained-release theophylline in patients with asthma/CVA and duration of asthma.

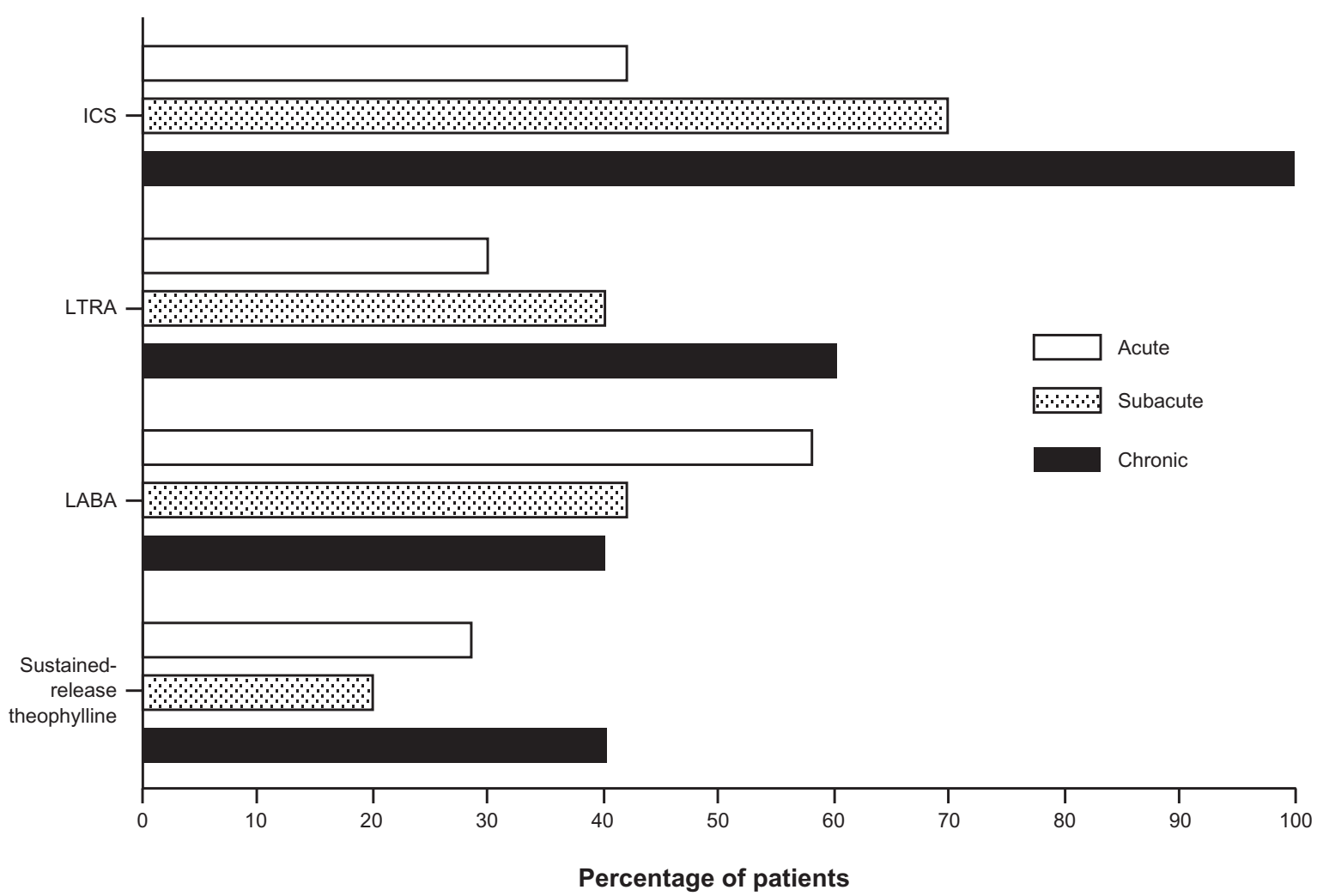

Figure 2 The percentage of antiasthmatic drug used by patients with asthma/CVA according to the duration of cough. The percentage of ICS use increased according to the duration of cough (acute, $42 \%$; subacute, $70 \%$; chronic, $100 \%$ ). The percentage of LTRA use also increased according to the duration of cough (acute, $30 \%$; subacute, $40 \%$; chronic, $60 \%$ ). The percentage of LABA use decreased according to the duration of cough (acute, $58 \%$; subacute, $42 \%$; chronic, $40 \%$ ). The percentages of sustained-release theophylline use did not correlate with the duration of asthma (acute, $40 \%$; subacute, $20 \%$; chronic, $29 \%$ ).

Abbreviations: CVA, cough variant asthma; ICS, inhaled corticosteroids; LABA, long-acting $\beta_{2}$-agonists; LTRA, leukotriene receptor antagonist. 


\section{Discussion}

Cough is the most frequent symptom for patients to visit clinic or hospital worldwide including Japan. ${ }^{1,3,4}$ Many diseases cause cough, but the distribution of diseases causing cough differs according to the duration of cough. The causes of acute or subacute cough tend to be infectious disease ${ }^{13}$ while the causes of chronic cough are likely noninfectious diseases. ${ }^{8}{ }^{814}$ Like previous studies, this study showed that the percentage of infectious diseases was high for causes of acute cough while the percentage of noninfectious diseases increased as the duration of cough became longer.

Causes of chronic cough differ among areas in Japan. Atopic cough and CVA are the most common causes of chronic cough. ${ }^{8,10}$ In this study, we found that bronchial asthma is the most common cause of chronic cough as well as of subacute cough. The second most common cause for chronic cough is CVA, the third is COPD, and the fourth is postinfectious cough. These differences between our results and others might come from the study period. In this study, we studied for a period of three months from April 1st to June 30th, 2007 while Fujimura and colleagues ${ }^{8}$ studied for about seven months and Shirahata and colleagues ${ }^{10}$ studied for over 12 months. Other reasons for these differences could be clinical background since those two studies might have excluded typical asthma and chronic obstructive pulmonary disease from patients complaining of chronic cough because they surveyed patients with no remarkable findings in chest $\mathrm{X}$-ray test and normal spirometry. There are subtle differences among areas of Japan in etiology of chronic cough. Fujimura and colleagues ${ }^{8}$ reported that CVA/asthma is the most common cause of chronic cough. Atopic cough is the second and sinobronchial syndrome is the third most common cause. Shirahata and colleagues ${ }^{10}$ and Fujimori and colleagues ${ }^{9}$ also reported that CVA is the most common causes of chronic cough. However, Shirahata and colleagues ${ }^{10}$ have reported that the second most common cause is nonspecific and the third most common cause is sinobronchial syndrome. Fujimori and colleagues reported that the second most common cause of the subacute is postinfectious and third most common is atopic cough. The reasons for these differences are not clear. However, confusion, or lack of consensus in disease entities which cause subacute and chronic cough might be one possible reason. Geographic issues such as distribution of general practitioners, local hospital generalists and respiratory physicians also affect the diagnosis of subacute and chronic cough. Therefore, causes of subacute and chronic cough in rural areas of Japan would be somewhat different from those in other studies.
Furthermore, diagnostic procedures or criteria might relate to the subtle differences in diagnosis of chronic cough. Fujimura and colleagues made differential diagnosis according to criteria recommended by the Japanese Cough Research Society. ${ }^{15}$ In their study, causes of cough were diagnosed symptoms, blood examinations, chest and sinus X-ray tests, examination of sputum eosinophilia, spirometry, capsaicin-induced cough sensitivity, bronchial responsiveness to methacholine and effectiveness of treatment for each disease. However, the precise diagnosis of CVA or atopic cough according to the Japanese Respiratory Society Guidelines $^{7}$ for general physicians is very difficult. In our study, spirometry was performed on more than half of the patients with CVA/asthma and the percentage of patients who had spirogram increased as to the duration of cough increased, though the percentage did not reach $100 \%$. On the other hand, blood examination is easy to carry out in general clinics and hospitals. The percentage of the patients who had the blood examination carried out including $\operatorname{IgE}$ and eosinophils increased according to the duration of cough However, the percentage of patients with IgE checked was smaller than those who were measured blood eosinophils. This result may indicate that the physicians measured blood white blood cells with differentiation to diagnose or exclude respiratory infectious disease. However, peripheral blood eosinophil as well as basophil percentage are important and useful for diagnosis of bronchial asthma. ${ }^{16}$ Furthermore, serum IgE is elevated in atopic and nonatopic asthma. ${ }^{17}$ The percentage of patients who checked sputum eosinophilia was about $50 \%$ of the patients who were diagnosed with asthma. The reason for this low percentage might be related to the patients' symptoms because this study was carried out for patients who had cough as a main symptom.

In this study, chest X-ray test was performed in almost all the patients with asthma among chronic cough and subacute cough groups. The diagnostic procedure to exclude diseases such as lung cancer, tuberculosis and interstitial pneumonia, is important because misdiagnosis and mistreatment of these diseases is critical. ${ }^{18-20}$ In this study, general physicians as well as pulmonary specialists must be careful to exclude these diseases. Furthermore, consequences of the exclusion of these diseases by chest X-ray test might lead to diagnosis of asthma/CVA. Induced sputum with hypertonic saline might be considered on patients with dry cough to show airway inflammation for diagnosis of asthma/CVA.

In this study, the treatment for asthma/CVA was analyzed. The percentage of ICS use in the patients with acute asthma/CVA was below $50 \%$, but this percentage increased 
according to the duration of cough and reached $100 \%$ in chronic asthma. Similar results were obtained in use of LTRA. In contrast to the use of ICS, the percentage of LABA use was over $50 \%$ but decreased according to the duration of cough. Since inhaled bronchodilator is used for the diagnosis and treatment of CVA, ${ }^{21}$ our results indicate that LABA might be used for acute asthma/CVA patients for treatment as well as for the diagnosis. On the other hand, the ICS might be used for the definite treatment for persistent symptoms such as subacute and chronic cough. ICS is most effective treatment for asthma and should be used in mild persistent, moderate and severe asthmatic patients ${ }^{22}$ or step 2 or more. ${ }^{23}$ Furthermore, Fujimura and colleagues have reported that nearly $30 \%$ of CVA patients develop typical asthma and no ICS use is one of the risks in developing typical asthma. ${ }^{24}$ Therefore, the treatment of chronic asthma/CVA in this rural area is compatible with international guidelines.

A number of limitations must be noted in this study. Firstly, the diagnosis of asthma or CVA has been made by each physician with laboratory findings as well as clinical symptoms. Therefore, diagnosis of asthma/CVA depends on each physician. Secondly, we asked the treatment for asthma/CVA according to each physician's diagnosis. This also includes diagnostic therapy. Since the Japanese guideline for diagnosis and management of cough $^{7}$ recommends using bronchodilator therapy for CVA for the initial diagnosis, it may influence the prevalence of LABA or sustained-release theophylline use. Finally, other disease such as COPD, GERD, or SBS is also diagnosed by each physician. However, our diagnostic procedures might be more practical in rural areas where sophisticated equipment is not readily available.

In the present study, it was difficult for the general physicians to use spirometry, sputum examination, measurement of capsaicin-induced cough sensitivity, and bronchial responsiveness to methacholine for diagnosis of asthma/CVA. However, most of the physicians were good at excluding the patients who were not asthma by checking X-ray test in almost all the patients who were diagnosed as asthma/CVA. Montnemery and colleagues have reported that general physicians are good at excluding patients who do not have asthma but not good at diagnosing patients who actually have asthma in Sweden. ${ }^{25}$ The precise diagnosis of asthma/CVA patients who complains of cough might be difficult. But clinical symptoms and laboratory tests such as peripheral blood examination, pulmonary function test, sputum examination and chest X-ray test are useful for asthma/CVA diagnosis. Simple diagnostic guidelines for asthma/CVA might be needed for general physicians.

\section{Acknowledgments}

We thank our subjects for their enthusiastic participation. We also thank The San-in Asthma Research Group members. None of the authors have any conflict of interest with the research reported in this manuscript.

The members of the San-In Asthma Research Group are as follows: Dr Akira Yamasaki, Dr Yasuyuki Hasegawa, Dr Masanari Watanabe, Dr Ryota Okazaki, Dr Naoto Burioka Dr Tadashi Igishi and Prof Eiji Shimizu at Multidisciplinary Internal Medicine, Tottori University; Prof. Takeshi Isobe at Department of Clinical Oncology and Respiratory Medicine, Shimane University: Dr Keiichi Hanaki, at Department of Pediatrics, Tottori University; Dr Takeshi Taketani, Dr Takeshi Kasai and Dr Hitoshi Sejima at Department of Pediatrics, Shimane University; Dr Kazuhiro Kato and Dr Kouji Fukutani at Department of Respiratory Medicine, San-in Rosai Hospital; Dr Yuji Sugimoto at Department of Internal Medicine, Tottori Prefecture Central Hospital; Dr Masahiro Kodani at Department of Internal Medicine, Tottori Red Cross Hospital; Dr Tatsuya Konishi at Department of Respiratory Medicine, Matsue City Hospital; Dr Yuji Kawasaki and Dr Hirokazu Tokuyasu at Department of Respiratory Medicine, Matsue Red Cross Hospital; Dr Toshikazu Ikeda at Department of Respiratory Medicine, National Organization Matsue Hospital; Dr Katsuyuki Tomita and Dr Hiroki Yajima at Department of Internal Medicine, Hakuai Hospital; Dr Masato Hirao at Department of Pediatrics, Tottori Red Cross Hospital; Dr Hiroshi Hayashibara at Department of Pediatrics, National Organization Yonago Medical Center; Dr Yasuhiro Fujii at Department of Respiratory Medicine, Saiseikai Sakaiminato General Hospital; Dr Tohru Hikita at Department of Allergy, Fujii Masao Memorial Hospital; Dr Daisuke Hori at Department of Respiratory Medicine, National Hospital Organization Hamada Medical Center; Dr Satoru Katayama at Department of Respiratory Medicine, Yoka Public Hospital: Dr Koichi Asai at Department of Pediatrics, Shimane Prefecture Hospital; Dr Yumi Negi and Yuuichi Mushimoto at Department of Pediatrics, Unnan General Hospital; Dr Takehisa Fujiwaki at Department of Pediatrics, Oochi General Hospital; Dr Masaru Tasaka at Department of Pediatrics, Tsuwano Kyozon Hospital; Dr Susumu Katsube at Katsube Clinic; Dr Yoshihide Nakamura at Nakamura Internal Medicine and Respiratory Clinic; Dr Hajime Horie at Horie Respiration Clinic; Dr Yuji Matsumoto at Matsumoto Clinic; Dr Takashi Matsuda at Matsuda Pediatric Clinic; Dr Masaaki Honda at Honda Clinic; Dr Sotarou Nogami at Nogami Clinic; Dr Kinya Motoda at Tottori Municipal Hospital, Dr Masato Horiuchi at Horiuchi Clinic; Dr Yuukou Yamane at Yamane Clinic; Dr Masaaki 
Mikami at Hosshouji Internal Clinic; Dr Tomoaki Koshoubu at Department of Internal Medicine, National Organization Yonago Medical Center; Dr Takashi Yanagawa at Department of Respiratory Medicine, National Hospital Organization Hamada Medical Center; Dr Kazuya Hirakawa at Shimane Prefecuture Preventive Medical Center; Dr Yoshimaro Yamamoto at Department of Internal Medicine, Tottori Prefecture Kousei Hospital; Dr Hiroyuki Yamamoto at Department of Internal Medicine, Saihaku Hosipital; Dr Naoto Kohno at Nichihara Kyozon Hospital; Dr Sumiko Takigawa at Sumire Pediatric Clinic; Noruki Haneda at Mototsune Pediatric Clinic; Dr Hiroshi Watanabe at Watanabe Pediatric and Ladies Clinic; Dr Isonori Senoh at Senoh Clinic; Dr Takaharu Ohie at Oda Municipal Hospital; Dr Yuichi Tagusa at Poyopoyo Clinic; Dr Yasuhisa Kajino at Department of Pediatrics, Masuda Red Cross Hospital; Dr Tadataka Hoshika at Department of Pediatrics, Tottori Prefecture Central Hospital; Dr Nobuo Ishitani at Ishitani Pediatric Clinic; Dr Teruo Okasora at Okasora Pediatric Clinic, Dr Junji Takagi at Department of Pediatrics, Gotsu Saiseikai General Hospital; Dr Seiko Yamane at Department of Pediatrics, Matsue Memorial Hospital; Dr Yasuo Nishino at Nishino Pediatric Clinic; Dr Hirohumi Urashima at Urashima Clinic.

\section{References}

1. Schappert SM, Nelson C. National Ambulatory Medical Care Survey: 1995-1996 summary. Vital Health Stat. 1999;13:i-vi, 1-122.

2. McCormick A, Fleming D, Charlton J. Morbidity Statistics From General Practice: Fourth national study 1991-1992. London, UK: The Stationery Office: 1995.

3. Bridges-Webb C, Dunstone MW. The Australian general practice morbidity and prescribing survey, 1969 to 1974 . 5. Aspects of morbidity: respiratory infections. Med J Aust. 1976;2:18-20.

4. Yamada T, Yoshimura M, Nagou N, et al. A Study on the outcomes of health problems (the concept of "episode of care") based on clinical statistics using the International Classification of Primary Care (ICPC). Jpn J Prim Care. 2000;23:213-219.

5. Irwin RS, Baumann MH, Bolser DC, et al. Diagnosis and management of cough executive summary: ACCP evidence-based clinical practice guidelines. Chest. 2006;129:1S-23S.

6. Morice AH, Fontana GA, Belvisi MG, et al; ERS guidelines on the assessment of cough. Eur Respir J. 2007;29:1256-1276.

7. Kohno S, Ishida T, Uchida Y, et al. Commitee for the Japanese Respiratory Society Guidelines for Management of Cough. The Japanese Respiratory Society guidelines for management of cough. Respirology. 2006;11:S135-S186.
8. Fujimura M, Abo M, Ogawa H, et al. Importance of atopic cough, cough variant asthma and sinobronchial syndrome as causes of chronic cough in the Hokuriku area of Japan. Respirology. 2005;10:201-207.

9. Fujimori K, Suzuki E, Arakawa M, Gejyo F. The diagnostic role of the methacholine inhalation challenge in adult patients with chronic persistent cough. Arerugi. 1999;48:713-718.

10. Shirahata K, Fujimoto K, Arioka H, Shouda R, Kudo K, Ikeda S. Prevalence and clinical features of cough variant asthma in a general internal medicine outpatient clinic in Japan. Respirology. 2005;10:354-358.

11. Levy ML, Fletcher M, Price DB, Hausen T, Halbert RJ, Yawn BP International Primary Care Respiratory Group (IPCRG) Guidelines: diagnosis of respiratory diseases in primary care. Prim Care Respir J. 2006;15:20-34.

12. Corrao WM, Braman SS, Irwin RS. Chronic cough as the sole presenting manifestation of bronchial asthma. N Engl J Med. 1979;300: 633-637.

13. Kwon NH, Oh MJ, Min TH, Lee BJ, Choi DC. Causes and clinical features of subacute cough. Chest. 2006;129:1142-1147.

14. Matsumoto H, Niimi A, Takemura M, et al. Prevalence and clinical manifestations of gastro-oesophageal reflux-associated chronic cough in the Japanese population. Cough. 2007;3:1.

15. Fujimura M, Uchida Y, Niimi A, et al. Guidelines for Diagnosis and Treatment of Chronic Cough, 2nd ed. Tokyo, Japan; Kita Media: 2003:1-35.

16. Lewis SA, Pavord ID, Stringer JR, Knox AJ, Weiss ST, Britton JR. The relation between peripheral blood leukocyte counts and respiratory symptoms, atropy, lung function, and airway responsiveness in adults. Chest. 2001;119:105-114.

17. Bousquet J, Coulomb Y, Arrendal H, Robinet-Levy M, Michel FB. Total serum IgE concentrations in adolescents and adults using the phadebas IgE PRIST technique. Allergy. 1982;37:397-406.

18. Calabrese F, Barrile D, Frison G, Nalesso A, Zuin R, Saetta M. An asthmatic patient with progressive lung dysfunction: a case of misdiagnosis. Lancet. 2006;368:814.

19. Novello A, Talenti E, Barbato A. Misdiagnosis of asthma. Two paradigmatic case reports. Minerva Pediatr. 1994;46:113-116.

20. Watanabe M, Tomita K, Burioka N, et al. Mucoepidermoid carcinoma of the trachea with airway hyperresponsiveness. Anticancer Res. 2000;20:1995-1997.

21. Irwin RS, French CT, Smyrnios NA, Curley FJ. Interpretation of positive results of a methacholine inhalation challenge and 1 week of inhaled bronchodilator use in diagnosing and treating cough-variant asthma. Arch Intern Med. 1997;157:1981-1987.

22. Ohta K, Tomioka K, Nishima M. Asthma Prevention and Management Guideline 2006, Japan. 1st ed. Tokyo, Japan: Guideline Committee on the Treatment and Management in Asthma, Kyowa Kikaku Inc: 2006.

23. National Heart Lung and Blood Institutes. Global strategy for asthma management and prevention. GINA report. National Institute of Health. 2006. Available from: http://www.ginathma.org/. Accessed 2006 Dec 21.

24. Fujimura M, Nishizawa Y, Nishitsuji M, Nomura S, Abo M, Ogawa H. Predictors for typical asthma onset from cough variant asthma. J Asthma. 2005;42:107-111.

25. Montnemery P, Hansson L, Lanke J, et al. Accuracy of a first diagnosis of asthma in primary health care. Fam Pract. 2002;19:365-368.

International Journal of General Medicine

\section{Publish your work in this journal}

The International Journal of General Medicine is an international, peer-reviewed open-access journal that focuses on general and internal medicine, pathogenesis, epidemiology, diagnosis, monitoring and treatment protocols. The journal is characterized by the rapid reporting of reviews, original research and clinical studies across all disease areas.

A key focus is the elucidation of disease processes and management protocols resulting in improved outcomes for the patient.The manuscript management system is completely online and includes a very quick and fair peer-review system. Visit http://www.dovepress.com/ testimonials.php to read real quotes from published authors. 